\title{
Practical Experience in Building an Agent System for Semantics-Based Provision and Selection of Grid Services
}

\author{
Gustaf Nimar $^{1}$, Vladimir Vlassov ${ }^{1}$, Kostantin Popov $^{2}$ \\ ${ }^{1}$ KTH/IMIT, Kista, Sweden. http: //www. imit.kth. se \\ ${ }^{2}$ SICS, Kista, Sweden. http://www. sics.se
}

\begin{abstract}
We present our practical experience in implementing an agent-based system for provision and selection of Grid services. The agents form a marketplace where services are offered and searched. Agents communicate semantic information about services using the Web service ontology (OWL-S). We describe our implementation that is built on Globus Toolkit 3 and utilizes the JADE agent framework and an off-the-shelf OWL-S toolkit. This combination of technologies can be used for more sophisticated agent-based services, such as e.g. automatic composition of services. We illustrate and evaluate our framework using a simple example, yet without loosing generality. We present preliminary evaluation results leaving stronger evaluation to future work. Our evaluation captures the relative costs of different stages during service provision and selection that allows detecting potential bottlenecks.
\end{abstract}

\section{Introduction and Related Work}

The Grid is envisioned as a global ubiquitous infrastructure that allows to treat all kinds of computer-related services as commodity - that can be described, located, purchased or leased, used, shared, etc. For specific needs services can be composed together forming new services called "virtual organizations" that deliver non-trivial qualities of service [9],[10]. The Grid is to be become large, decentralized and heterogeneous.

The scale and decentralization of the Grid implies that Grid clients and services can possess only partial information about the Grid. Moreover, access to some information can be restricted due to security. Finally, the Grid is volatile, thus any information accumulated about it is inherently imprecise. The agent-based approach (e.g. [20],[21]) is generally considered to facilitate system development for such environments [13], and its virtue for the Grid is well recognized [8][6],. An agent in a multi-agent system (MAS) serves a specific role; it is situated in a particular environment; it has complete control over its own state and behaviour; it can communicate with other agents, and its internal and external behaviors are flexible depending on its state and environment. Agent-based software engineering can be used for building decentralized intelligent applications [16] on the Grid. It can also be used for enhancing the Grid infrastructure itself, providing e.g. knowledge-based information services, semantic service description etc. [6] that will utilize and complement the present day Grid infrastructure [8]. 
Services in a Grid's Virtual Organization, similar to agents in MAS, need flexible communication [8], [6]: its form and content changes following the evolution of the agent's state and environment, as well as the system structure can change over time. Communication using semantic, self-explanatory information addresses the problem [6], as agents can exchange both syntactic data and the knowledge domain in which the data is to be interpreted.

The use of semantic information for agent communication is already being standardized by FIPA [7] (the primary standardization body in MAS). In particular, in the Abstract Service Architecture the agent's "service description" contains semantic information [5]. W3C coined the notion of "semantic web" [1] that addresses the issues of expressing meaning, knowledge representation and usage, ontologies, and agent-based construction of Web services. These developments are supported by the W3C's endorsed work on Resource Description Framework (RDF), Web Ontology Language (OWL) and Web service ontology (OWL-S) [19]. RDF is a data model for entities and relations between them. It provides a simple semantics for this model and a representation schema in XML syntax. OWL extends RDF and can be used to explicitly represent the meaning of entities in vocabularies and the relationships between those entities. OWL-S defines a standard ontology shared by Web services.

In this paper we present our practical experience in integrating the state-of-the-art agent and semantic-web technologies in a Grid service based on the current release of the Globus Toolkit 3 [11]. We believe our results will remain valid for the forthcoming GT4, as we do not crucially depend on any particular aspect of GT3's OGSI [18]. To the best of our knowledge, this is the first report describing design, implementation and analysis of such a Grid service.

We focus our study on use of agents and semantic information for service provision and selection. Clients need to locate suitable services, whereas providers can impose constraints on how their services can be used. This is a real-world example where the Grid's scale, dynamism and heterogeneity encourage an agentbased design. Clients are represented by service selection agents that work on behalf of clients and guard their interests. Service providers are represented by service provision agents that provision services according to providers' interests. Both agents interact to achieve a mutual agreement. Each client or provider should be represented by its own agent because the agent can contain confidential information.

Provision and selection agents have to communicate with semantic information for the sake of flexibility. First of all, a system for provision and selection of Grid services has to handle arbitrary types of services. Next, there should be an expressive language for specification of client's needs that might be difficult to translate into a rigid language for agent communication. Finally, negotiation may take several steps that can be difficult to express in a rigid communication language.

Our prototype illustrates the issues in building such a system. Our limitations are that we have used a simple query language at the client's end (a WSDL document the same type of description used at provider's end), and we have implemented a very simple matching algorithm. These limitations, however, are encapsulated in particular components in our implementation, and the performance analysis factorizes away the performance properties of those components.

Surveys on Multi-Agent Systems [16, 13] show, in particular, how agents can be used for resource brokering, workflow management and planning. Research is active 
in particular in the fields of negotiation, communication languages, ontologies and scalability issues; this research is reflected in numerous publications in dedicated tracks of International Joint Conferences on Autonomous Agents and Multi-agent Systems (AAMAS). In the context of Grid, agents are in particular used for resource brokering [4] and for scientific computing and workflow management [14, 15, 3]. Following the semantic web, the notion of "Semantic Grid" was coined $[8,6]$. Concerning service provision and discovery, work is done on semantic matching e.g. $[2,17]$ which however does not consider the use of agents and system design and implementation issues. Ontology-driven resource discovery for Grids is also considered in [12] which adopts the peer-to-peer approach.

\section{System Architecture}

The overall system architecture is structured as a marketplace where agents handle both provision and selection of Grid services (as seen in Fig.1). In order to select a service we must find a way to provision them first. This includes finding a way to describe Grid services and making these descriptions available for agents handling the service providing part in a negotiation, i.e. a Service Provision Agent (SPA).

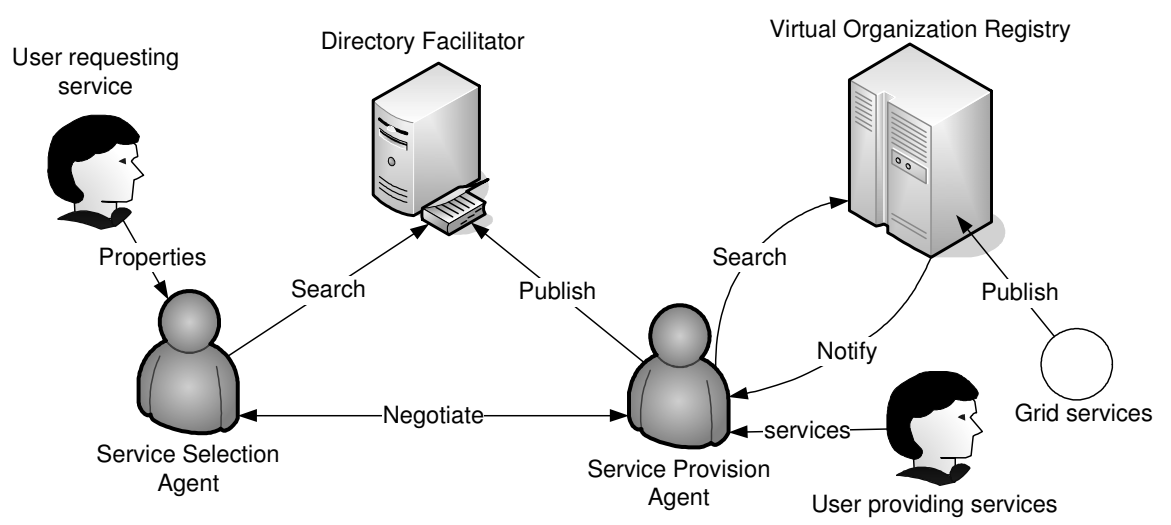

Fig. 1. Architecture of the Agent-Based System for Grid Service Provision and Selection

Services to be provisioned are assigned to an SPA in the system. An SPA can be assigned a single service or several services registered at a VORegistry, which is a simple registry service included in Globus Toolkit 3. To be found by Service Selection Agents (SSA), SPAs publish themselves at a Directory Facilitator (a predefined agent holding a directory where other agents can publish themselves and search for others). A user requesting a service specifies its requirements to an SSA by providing a service description in the form of a WSDL document and additional semantic data. These are transformed into a sample service in a supported ontology. Then the SSA starts to negotiate with the agents provisioning services. Each SPA involved in the negotiation searches its local storage for services matching the requested service and returns the best match. 


\subsection{Definition of Services}

In our system we assume that a service to be provisioned must be initially defined in GWSDL as required by GT3 (OGSI). To improve precision of selection, the service definition should also provide semantic data such as its properties, capabilities, and taxonomy. These data are defined in Service Data Elements (SDEs) of the GWSDL service definition. Once a service has been deployed in a Grid service container and (optionally) registered at VORegistry, it can be assigned to an SPA which will combine the definition elements into an ontology object.

When a user (it can be a program) wants to select a Grid service using an SSA, it specifies requirements to the service. The specification contains a GWSDL definition including SDEs that define requirements or/and constraints to be matched with properties and capabilities of offered services. The definition does not need to be complete as it is used as "a pattern" in selecting real services offered at a marketplace.

In the system, agents use service definitions in OWL-S, which are obtained by translating initial WSDL descriptions into OWL-S definitions. The semantic data defined in SDEs are placed to service profiles in OWL-S descriptions.

\subsection{Provision of Service}

In order to provision services, a user (it can be a program using the framework) instantiates a Service Provision Agent (SPA) and assigns either a single service or services of one or several Virtual Organizations (VOs) to the agent.

A sequence diagram of communication required to assign resources of a $\mathrm{VO}$ to an SPA is shown in Fig.2(a). If the SPA assigned a VO, it fetches descriptions of all the VO services from the VORegistry. Then SPA constructs a description in a supported ontology using the WSDL document (with additional SDEs) of each of services found in the registry. Finally, the SPA publishes itself at the Directory Facilitator.

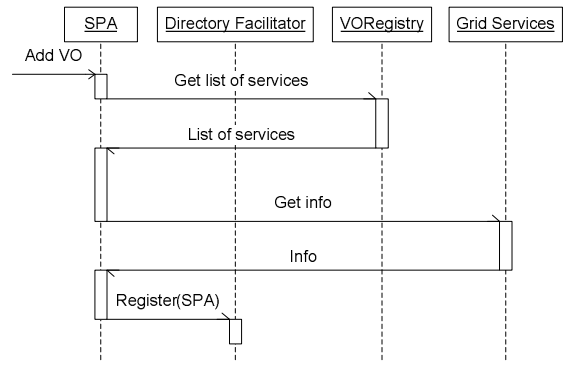

(a) Assigning a VO to an SPA

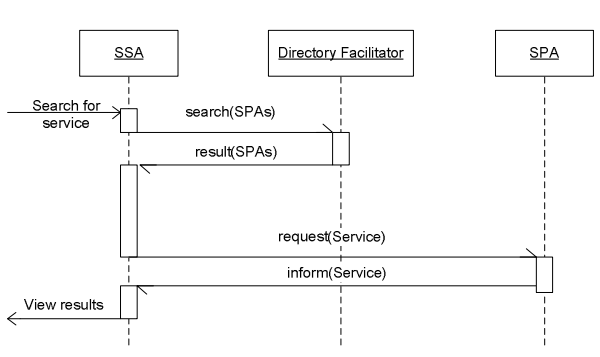

(b) Selecting a service

Fig. 2. Communication sequence diagrams

\subsection{Selection of Service}

The goal of an SSA is to select the service best suited for a user's requirements. The SSA translates the user's requirements into a description of a synthetic service in a 
chosen ontology. On a request of the SSA, this description is compared by SPAs with the descriptions of provisioned services in the same ontology. The SSA then can select a best match among services offered by SPAs.

The communication sequence diagram of service selection is shown in Fig.2(b). When a client initiates a search for a service using an SSA, the agent first downloads a list of available SPAs from the Directory Facilitator and selects the SPAs to interact with (based on the supported ontologies). The SSA sends the formalized user's query to the selected SPAs in a FIPA ACL request message. Each SPA searches its storages for matching services and sends search results back to the SSA in an FIPA ACL inform message. Upon receiving the descriptions from SPAs, the SSA can further search the descriptions for a best match or/and presents the descriptions to the client.

A system that provides service selection should support different matching algorithms and different search strategies used by agents. In order for a matching algorithm to achieve tolerable results, it should comprise the following properties: encourage the requestors and the providers of services to be detailed in their descriptions of services; include semantic matching of inputs and outputs; and allowing for prioritizing the search categories. In the example presented later in the paper, we describe one of possible algorithms comprising these features. Study of matching algorithms is out of the scope of this paper.

\subsection{Information Flow}

In the system, agents communicate different forms of service descriptions as shown in Fig.3. A service description can enter the system in two different ways: as a service description of a new synthetic service to be searched by an SSA; or as a description (along with the associated semantic SDEs) of a real service provisioned by an SPA.

Let us first consider the case when a definition of a synthetic service is created by a client to be used as a pattern in service selection. The WSDL-definition of service operations along with SDEs containing semantic data are represented in the system as set of objects (see Fig.3) which are converted into a single service description object in a preferable ontology by a WSDL-2-ontology translator. Once the service description object in the ontology has been constructed it is transformed into an XML document by a service description writer to be sent in the ACL message to an SPA. The receiving SPA extracts the XML document which is transformed to an ontology object by a service description reader. The object is compared with descriptions of provisioned services by a Service Matcher. The Service Matcher returns the best matching object(s) which is then transformed to an XML document by the service description writer. The XML document is sent back to the SSA in the ACL message. The document is extracted into an ontology object by the service description reader.

On the SPA side (Fig.3), the WSDL document along with associated SDEs is fetched by the SPA using the Grid Service Handle (GSH). The WSDL definition of non-standard (user-defined) operations is represented by one or several objects created by WSDL service creator. These objects along with the SDEs are translated by the WSDL-2-Ontology translator into a description object in the ontology. The object is stored in the local storage. 


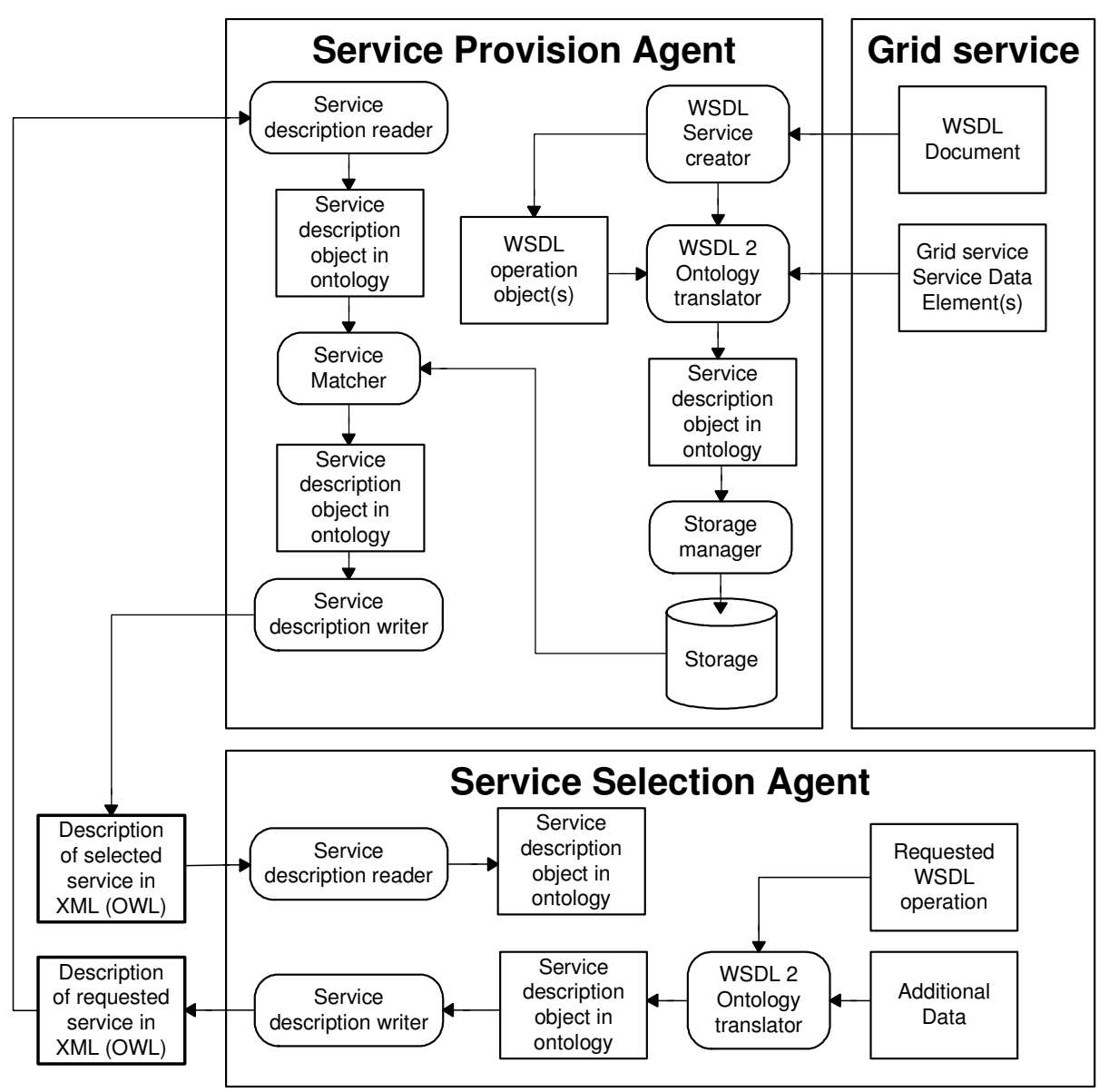

Fig. 3. The information flow in the system

\section{A System Prototype}

This section describes a prototype implementation of the agent-based framework for Grid service provision and selection described in the previous section. Due to the fact that both JADE (Java Agent DEvelopment) framework and GT3 (Globus Toolkit) used for implementation support development in Java, our system prototype has been implemented in Java using the following software platforms and development tools: JADE 3.1 [23], GT 3.2 [24], Java SDK 1.4.2 [25]. In addition, we used OWL-S-1.0.1 [22] that is a Java API for reading, executing and writing OWL-S. The OWL-S-1.0.1 API has been revised in order to support the serviceCategory (including taxonomy) located in the ServiceProfile of a service description. The revision affected the reader and the writer of XML documents as well as the representation of ontology objects (in OWL-S 1.0). We also used Jdom 1.0 [26] that is a Java API for reading, 
manipulation, and writing Java representation of XML. As the system prototype was implemented before Java SDK 1.5 was released, we did not use Web Service support in Java SDK 1.5. When developing experimental Grid services we also used Eclipse 3.0 [27] with the additional Globus Toolkit Plug-in for Eclipse 0.2.0 [28].

The classes and interfaces of the system prototype have been organized in the following five packages: agents, content, grid, matcher and storage. The agents package holds implementations of agents based on the JADE platform. The content package includes classes for managing the content carried in the ACL messages together with classes used when working with WSDL documents. The package has been divided into three sub packages: lang (classes to work with the content languages included in the ACL messages), owls (classes to manage the OWL-S ontology), and wsdl (interfaces and classes of the rewritten OWL-S API mentioned above). The grid package includes classes that communicate directly with Grid services. The package, in particular, contains methods for extracting Service Data elements given a GSH. The matcher package includes classes for matching the requested service description expressed in OWL-S against the ones being advertised in the same ontology. In the matching algorithm described earlier, matching of input and output parameters types is based on the URI of the types. This means that providers and requestors of services can define their own types. The classes responsible for storing descriptions of the services at a Service Provision Agent are grouped in the storage package. The system prototype allows the storage to be implemented using various technologies, e.g. databases or classes of the Java collection framework.

\subsection{Implementing a Service Provision Agent}

The SPA is implemented by the class ServiceProvisionAgent which extends the JADE Agent class. The SPA should include functionality for assigning services and VOs. The ServiceProvisionAgent also includes functionality for performing service matching. The actions of each agent are based on JADE behaviours. The behaviours needed to cover the functionality required of an SPA are listed in Table 1.

The first thing to do when assigning a new service to an SPA is to add addService behaviour. If the agent isn't already registered at the Directory Facilitator it will also add RegisterSPA behaviour and finally start listening for incoming requests, i.e. adding ListenForReq behaviour.

Table 1. Behaviours of the Service Provision Agent

\begin{tabular}{|c|c|}
\hline Behaviour & Description \\
\hline addService & $\begin{array}{l}\text { Assigns a service to an SPA given a GSH. Using the WSDL document } \\
\text { representing the service, all non-standard Grid service operations are translated } \\
\text { into service descriptions. The descriptions are then handed to the local storage. } \\
\text { Fetches the GSHs of all the services located in the registry. Each of the services } \\
\text { is added to the SPA's storage using the addService behaviour. }\end{array}$ \\
\hline ListenForReq & $\begin{array}{l}\text { A cyclic behaviour that listens for incoming requests. If a message is received it } \\
\text { will be parsed and the right action will be taken. }\end{array}$ \\
\hline RegisterSPA & Registers itself at the Directory Facilitator. \\
\hline SearchAndResponse & Searches the local storage for the requested service and sends the result back. \\
\hline
\end{tabular}




\subsection{Implementing a Service Selection Agent}

The SSA is implemented by the class ServiceSelectionAgent that extends the JADE Agent class. Like the ServiceProvisionAgent, invocation of the selection agent is supported by the platform. The behaviours used by the SSA are listed in Table 2 .

One interesting method of the ServiceSelectionAgent class is the search method that initializes a search for a service. Every search is identified with a unique identification number. The first behaviour executed in the search is the GetSPAs, which fetches a list of available SPAs. Then a parallel behaviour is invoked, executing one or more SearchSPA behaviours in parallel. Each of the SearchSPA behaviours sends a request to one of the SPAs. Finally a second parallel behaviour is executed including both a receive behaviour (collecting results) and a Timeout behaviour (terminating the search after the given timeout). The Timeout behaviour will also sort the collected results finding the best suited service.

Table 2. Behaviours of the Service Selection Agent

\begin{tabular}{l|l}
\hline Behaviour & \multicolumn{1}{|c}{ Description } \\
\hline GetSPAs & $\begin{array}{l}\text { Searches the Directory Facilitator for available SPAs. } \\
\text { A cyclic behaviour that listens for incoming result messages. A received message is } \\
\text { parsed and if it contains search results it's stored in a result vector. The behaviour can } \\
\text { be terminated by calling the setDone method. } \\
\text { SearchSPA }\end{array}$ \\
$\begin{array}{l}\text { Searches a given SPA for the requested services, i.e. sends an ACL request with a } \\
\text { findService Action. } \\
\text { Behaviour that sleeps for a while, wakes up after a given timeout, and terminates the } \\
\text { collection of search results and starts evaluating the results. }\end{array}$ \\
\hline
\end{tabular}

\subsection{Grid Service Extension}

Properties that cannot be defined in a WSDL document are defined in Service Data Elements. The ontology currently supported by the prototype OWL-S, includes such properties. Some are used in our matching algorithm, e.g. the data fields of the Service Category. In the proposed solution we created an SDE called OwlsDataType holding the necessary properties (Fig.4). The Service Data type can be imported into any Grid service description using the import element.

\section{An Example: Sending Messages to Mobile Phones}

To illustrate a typical usage of our framework, consider a simple example. Assume four mobile operators providing services in sending information (SMS messages, MMS messages, ring tones, and images) to mobile phones as Grid services. One of the main reasons why Grid services are well-suited for these kinds of services is the ability to define additional service data which in our case carries semantic data. Furthermore, it might be convenient to charge a client for all messages sent within some period of time by associating an instance of a service with a client instead of charging each message sent by means of a stateless Web service. 


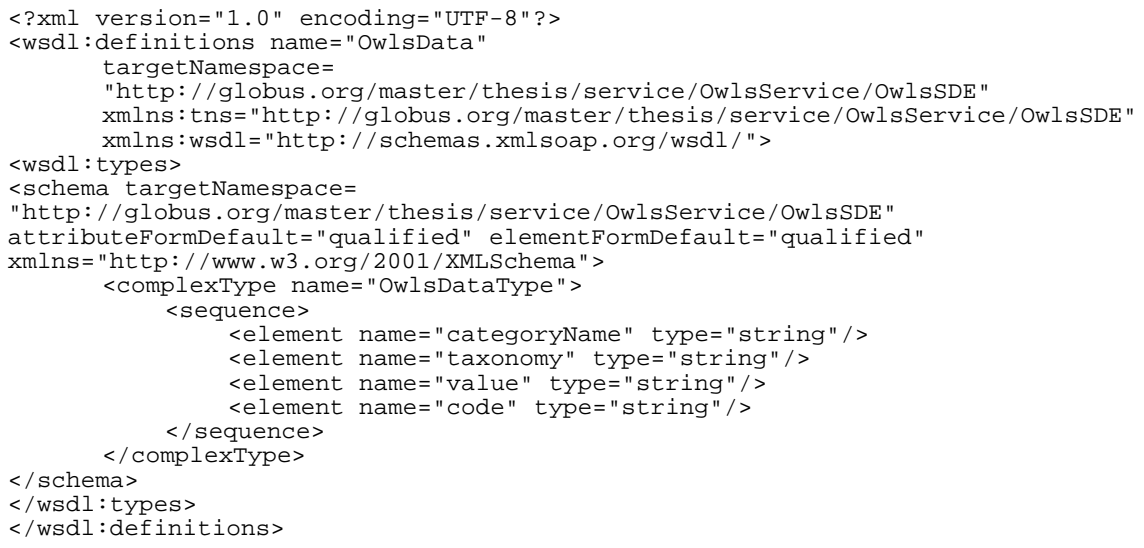

Fig. 4. The Service Data Type OwlsDataType

Assume each mobile operator is represented by an SPA. A Grid service offered by an operator is described in WSDL with additional Service Data (based on taxonomy) and converted into the OWL-S 1.0 service description. The service is assigned to the operator's SPA which registers at the Directory Facilitator once service descriptions are stored locally. The services provisioned by each of the SPAs are listed in Table 3.

Table 3. The operator's SPAs and their services

\begin{tabular}{|c|c|c|}
\hline Agent & Operations (WSDL pseudo code) & Service Category $(\mathrm{SDE})$ \\
\hline \multirow[t]{4}{*}{ SPA1 } & sendSMS (msg:String, num:long) $->$ (status:bool) & \multirow{4}{*}{$\begin{array}{l}\text { categoryName: } \\
\text { currency } \\
\text { code: LESSTHAN } \\
\text { taxonomy: sek } \\
\text { value: } 1.3\end{array}$} \\
\hline & sendMMS (msg:MMS, num:long) $->$ (status:bool) & \\
\hline & sendRingTone (tone:int, num:long) $->$ (status:bool) & \\
\hline & $\begin{array}{l}\text { sendPicture (picture:int, num:long) } \rightarrow> \\
\text { (status:bool) }\end{array}$ & \\
\hline \multirow[t]{4}{*}{ SPA2 } & SMSSender (msg:String, num:int) $->$ void & \multirow{4}{*}{$\begin{array}{l}\text { categoryName: } \\
\text { currency } \\
\text { code: LESSTHAN } \\
\text { taxonomy: sek } \\
\text { value: } 1.4\end{array}$} \\
\hline & MMSSender (msg:MMS, num:int) $->$ void & \\
\hline & RingToneSender (tone:int, num:int) $\rightarrow$ void & \\
\hline & PictureSender (pic:int, num:int)-> void & \\
\hline \multirow{4}{*}{ SPA3 } & sendSMS (msg:String, num:long) $\rightarrow$ (status:bool) & \multirow{4}{*}{$\begin{array}{l}\text { categoryName: } \\
\text { currency } \\
\text { code: LESSTHAN } \\
\text { taxonomy: sek } \\
\text { value: } 1.6\end{array}$} \\
\hline & sendMMS (msg: MMS, num:long) -> (status:bool) & \\
\hline & sendRingTone (tone: int, num:long) $->$ (status:bool) & \\
\hline & $\begin{array}{l}\text { sendPicture (picture:int, num:long)-> } \\
\text { (status:bool) }\end{array}$ & \\
\hline \multirow[t]{4}{*}{ SPA4 } & $\begin{array}{l}\text { birthdaysMS (message: string, num:long, } \\
\text { time: dateTime) } \rightarrow \text { (status: bool) }\end{array}$ & \multirow{4}{*}{$\begin{array}{l}\text { categoryName: } \\
\text { currency } \\
\text { code: LESSTHAN } \\
\text { taxonomy: sek } \\
\text { value: } 1.3\end{array}$} \\
\hline & $\begin{array}{l}\text { birthdayMMS (message:MMS, num:long, time: dateTime) } \\
\rightarrow \text { (status:bool) }\end{array}$ & \\
\hline & $\begin{array}{l}\text { birthdayRingTone (tone: int, num:long, } \\
\text { time: dateTime)-> (status:bool) }\end{array}$ & \\
\hline & $\begin{array}{l}\text { birthdayPicture (pic:int, num:long, time:dateTim } \\
\rightarrow \text { (status:bool) }\end{array}$ & \\
\hline
\end{tabular}

Assume that a Service Selection Agent is to find a service sending SMSs that has an operation similar to SMS(msg:string, number:long) -> (status: boolean) that costs less than 1.5 SEK. The SSA will transform a WSDL definition of the required service to an OWL-S description object that is sent to SPAs in a FIPA ACL request message.

For simplicity, without loosing generality, we use a simple matching algorithm shown in Fig. 5. The algorithm matches a requested service against all the services 
provisioned by the SPA. Each provisioned service is assigned an integer score - the higher the score is, the better the service suits. The service that receives the highest score is the one returned to the requestor (which will encourage requestors and providers of services to give detailed descriptions of their services). The score is based on a weighted addition of the results of four different comparison methods. The weights make it possible for prioritizing between the methods. The first method matches the inputs parameters; the second the output parameters; the third the names of the services; and finally the fourth method matches the taxonomy.

According to the matching algorithm, the best match for the requested service will be the service offered by the first provision agent SPA1 for 1.3 SEK.

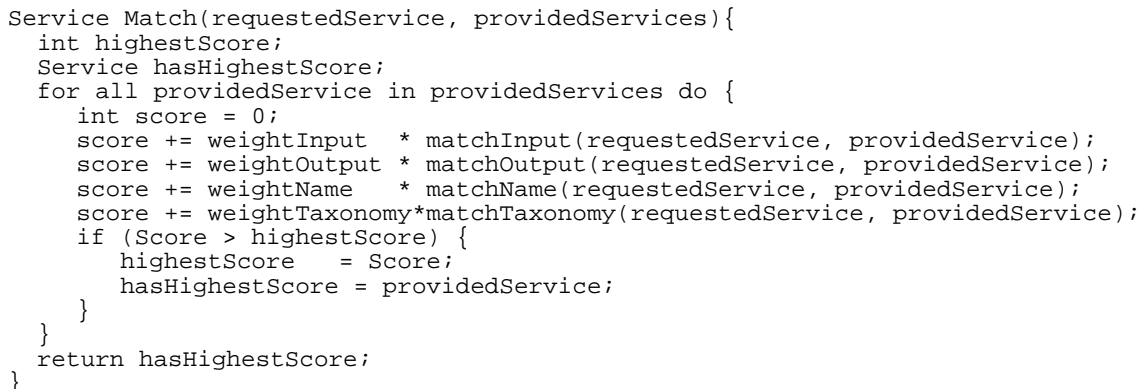

Fig. 5. The Service Matching Algorithm

\section{Preliminary Evaluation of the Framework}

In this paper, we present results of preliminary evaluation of the ontology-enabled agent-based framework rather than the system prototype based on the framework. We leave for future work more detailed evaluation of the framework and evaluation of performance, scalability and reliability of the system prototype. In order to determine potential bottlenecks in the framework we measured the time consumed by different parts of the system prototype.

In order to validate and evaluate the framework, we ran the system on an AMD Athlon 1800+ under Windows XP. To run the agent platform and a Grid container, we used Java SDK 1.4.2, JADE .3.1 [23], a modified version of OWL-S-1.0.1 [22], Jdom [26], and GT3.2 [11].

In the first series of experiments, we measured the time consumed by different parts of the system when an SPA executes the service provision behaviour, i.e. creates an OWL-S service description object given the Grid service's WSDL document and additional Service Data. The overall time of provisioning of four services described in the above example was $1209 \mathrm{~ms}$. Our experiments showed that when provisioning service, the part of the system interacting with the Grid container (in our case an Apache server) consumed about $90 \%$ of the total time. In particular, measurements showed that the Apache WSDL parser was the most time consuming element. Experiments also showed that fetching service data consumed $4,5 \%$ of the total 
provision time; storing of four services (i.e. merging WSDLService objects and corresponding additional Service Data into service descriptions in the preferable ontology) consumed from $0,2 \%$ to $6 \%$ per service.

In the second series of experiments, we measured time spent in different parts of the system when an SSA selects services provided by several SPAs. The total wall clock time for selection among four services was $813 \mathrm{~ms}$. The selection time is highly dependent on the timeout value (the time waiting for results) set for the SSA when it initiates a search. In our experiments, the timeout value was set to $500 \mathrm{~ms}$. When the SSA timeouts it sorts the incoming results and selects the best suited service. Measurements showed that the JADE agent platform itself consumed most (about $60 \%$ ) of the overall selection time. Apart for the platform the conversion of ontology objects into XML documents, and vice versa, were also time consuming $(18 \%$ consumed for writing and $22 \%$ for reading XML documents).

Apart from measuring time consumption, we evaluated memory usage in the system prototype. The results show that a single service description consumes $80 \%$ of the total memory usage, when kept in a collection object (e.g. Vector) in the main memory, compared to an agent of each kind. One solution when it comes to large amount of services descriptions would be to use databases (or text files), whereas object collections can be used to provide caching of descriptions in the object memory. Considering this issue is a subject for future work.

\section{Conclusions and Future Work}

We have presented our approach and a practical, hands-on, experience in creating an agent-based system for service provision and selection in Grids using semantic Grid service descriptions. A system is based on using GWSDL and OWL-S languages to define service operations and to provide additional semantic data (properties and capabilities, or constraints and requirements) on Grid services to be provisioned by Service Provision Agents (SPA) and/or to be searched by Service Selection Agents (SSA). The system is based on the OWL-S ontology that allows defining properties and capabilities of services. Initially, semantic information is provided in service data elements of WSDL service descriptions, which are then translated into OWL-S descriptions. An SPA may use different matching algorithms to match its service properties and capabilities against requirements and constraints given by an SSA.

A system prototype was implemented using available programming platforms and environments, namely the JADE agent platform, the Globus Toolkit (GT3), the OWLS-1.0.1 [22] Java API for working with OWL-S (in particular, WSDL-to-OWL-S conversion), and the Jdom 1.0 [26] Java API for working with XML.

Our future work includes porting the system prototype to GT4; stronger evaluation of performance and scalabililty of the system prototype and the framework; considering different matching algorithms; experimenting with information stored in Service Data Elements presented in other (different) ontology; considering security aspects. Our future plans also include providing support for service composition. 


\section{References}

[1] T. Berners-Lee, J. Hendler, and O. Lassila. The semantic web. Scientific American, May 2001.

[2] J. Brooke, D. Fellows, K. Garwood, and C.A. Goble. Semantic matching of grid resource descriptions. In Proceedings of The Second European Across Grids Conference, Nicosia, Cyprus, 2004.

[3] J. Cao, S.A. Jarvis, and S. Saini. ARMS: An agent-based resource management system for grid computing. Scientific Programming, 10(2):135-148, 2002.

[4] K. Czajkowski, I. Foster, and C. Kesselman. Resource and service management. In Foster and Kesselman [10].

[5] J. Dale and M. Lyell. Towards an abstract service architecture for multi-agent systems. In Workshop on Challenges in Open Agent Systems'03, 2003.

[6] D. de Roure, N. R. Jennings, and N. Shadbolt. The semantic Grid: Past, present and future. Proceedings of the IEEE, 93, 2005.

[7] Foundation for Intelligent Physical Agents (FIPA). www.fipa.org.

[8] I. Foster, N.R. Jennings, and C. Kesselman. Brain meets brawn: Why grid and agents need each other. In Third International Joint Conference on Autonomous Agents and Multiagent Systems (AAMAS'04), New York, USA, July 2004. IEEE.

[9] I. Foster, C. Kesselman, and S. Tuecke. The anatomy of the Grid: Enabling scalable virtual organizations. International J. of Supercomputer Applications, 15(3), 2001.

[10] Ian Foster and Carl Kesselman, editors. The Grid 2: Blueprint for a New Computing Infrastructure. Morgan Kaufmann, 2nd edition, 2003.

[11] The Globus Alliance. www.globus.org.

[12] F. Heine and M. Hovestadt. Towards ontology-driven P2P Grid resource discovery. In 5th IEEE/ACM International Workshop on Grid Computing, November 2004.

[13] N.R. Jennings. An agent-based approach for building complex software systems. Communications of the ACM, 44(4):35-41, 2001.

[14] K. Kumar. An agent-based Grid flow management framework (GFMF). Presented at GlobusWorld 2004, 2004.

[15] Moreau et al. On the use of agents in bioinformatics Grid. In CCGRID'03: $3^{\text {rd }}$ International Symposium on Cluster Computing and the Grid. IEEE, 2003.

[16] Katia P. Sycara. Multiagent systems. AI Magazine, 19(2), summer 1998.

[17] H. Tangmunarunkit, S. Decker, and C. Kesselman. Ontology-based resource matching in the Grid - the Grid meets the semantic web. In 2nd International International Semantic Web Conference (ISWC2003), pages 706-721, October 2003.

[18] Tuecke et al. Open grid services infrastructure (OGSI) version 1.0. Global Grid Forum OGSI Working Group, June 272003.

[19] World Wide Web Consortium (W3C). www.w3c.org.

[20] M. Wooldridge. Agent-based software engineering. IEEE Proceedings of Software Engineering, 144:26-37, 1997.

[21] M. Wooldridge. Introduction to MultiAgent Systems. John Wiley \& Sons, 2002.

[22] Evren Sirin. OWL-S API. http://www.mindswap.org/2004/owl-s/api/

[23] Telecom Italia Lab. Jade 3.1. http://jade.tilab.com/

[24] The Globus Alliance. Globus Toolkit 3.2. http://www-unix.globus.org/toolkit/

[25] Sun Microsystems. Java 2 Platform, Standard Edition (J2SE). http://java.sun.com/j2se/

[26] The JDOM TM Project. Jdom 1.0. http://jdom.org/

[27] Eclipse Foundation. Eclipse 3.0. http://www.eclipse.org/

[28] B. Sotomayor, M. López and T. Sánchez. A Globus Toolkit Plug-in for Eclipse. http://gt3ide.sourceforge.net 